\title{
Study on NTC Electrical Behavior of Carbon Black / PVC Composite
}

\author{
Xiuqi Liu ${ }^{1, a}$, Heqin Xing ${ }^{2, b}$, Lili Zhao ${ }^{1, ~ a}$, Xishuang Chen ${ }^{1, \text { a }}$ \\ ${ }^{1}$ College of Food Science and Engineering, Jilin Agricultural University, Changchun, Jilin, 130118, \\ China \\ ${ }^{2}$ College of Quartermaster Technology, Jilin University, Changchun, Jilin, 130062, China \\ aliuxiuqi2004@yahoo.com.cn, bxinghq@jlu.edu.cn
}

Keywords: Composite, Negative temperature coefficient (NTC), Carbon black, Polyvinyl chloride (PVC).

\begin{abstract}
In our study, Carbon Black / PVC NTC composites were prepared by conventional melt-mixing technique, the resistivity-temperature characteristic of the composites were studied. The results show that the composite possess a lower percolation threshold, a notable negative temperature coefficient (NTC). the volume resistivity was reduced, when carbon black filling rate increased to a characteristic value, the resistivity reached 5 to 10 orders of magnitude; from the SEM photograph of composite, the dark and light areas were relatively distributed, the dispersion effect of the carbon black was good.
\end{abstract}

\section{Introduction}

As one of the five most universal plastic, PVC has been widely used; it is easily obtained and low-cost and has rather mature manufacturing technique. Otherwise PVC has good incombustibility, anti-chemical corrosion, high tension and electric insulativity. The theories of PVC modification are abundant due to the widely studies [1-3]. At present, PVC conductive materials are becoming important products in our common life.

For physics property, high thermal expansion, cushioning ability, and conductivity are mainly utilized. The electrical properties of polymer are mainly resistivity-temperature characteristic (PTC effect and NTC effect) and switching characteristic [4-5]. PVC conductive composite as the NTC device, it can be used as a protective sheath of the cable and detected for a cable "hot spots", the circuit system can be prevent damage to the people and equipment.

\section{Experimental}

Materials. PVC: S-III, Daqing Petrochemical Plastic Factory, China; Conductive carbon black: HG-1P, Huaguang Chemical Factory, China; Dioctyl phthalate (DOP): Shanghai Chemical Co., China; Barium stearate: Shanghai Chemical Co., China; Liquid olefin: Zhenhai Institute of Applied Chemistry, China.

Preparation of Carbon Black / PVC Composite. Carbon black, PVC, DOP and other additives were mixed uniform, placed the blend in a vacuum oven for 1 hour at $80{ }^{\circ} \mathrm{C}$. And then the blend was melting blended on a closed mill for $20 \mathrm{~min}$ at $125^{\circ} \mathrm{C}$, after that the composite was taken out and masticated on an open mill for $5 \mathrm{~min}$ and stabilized to remove the strain overnight ( $24 \mathrm{~h}$ if possible) before being vulcanization.

Measurement of Volume Resistivity ( $\rho)$. Volume resistance R of the direction of the specimen thickness was tested with a second electrode. The both ends of sample were coated with conductive silver glue to ensure good contact of the sample with copper electrodes. VC-9808 digital multimeter was used for measure resistance, volume resistivity was converted using the following expression:

$$
\mathrm{P}=\mathrm{R} \times \mathrm{S} / \mathrm{L}(\Omega \cdot \mathrm{cm})
$$

R: volume resistivity $(\Omega)$; S: surface area of specimen $\left(\mathrm{cm}^{2}\right)$; L: sample thickness $(\mathrm{cm})$ 
Measurement of Volume Resistivity - Temperature (p-t). The sample was placed in a thermostatic oven, the heating and cooling rate was $2{ }^{\circ} \mathrm{C} / \mathrm{min}$, every $5 \mathrm{~min}$, resistance - temperature changes was record, make the volume resistivity - temperature $(\rho-T)$ curve.

\section{Results and Discussion}

Relationship of Resistivity and Carbon Black Fill Rate. The relationship of room temperature resistivity $\rho$ and the $\mathrm{CB}$ mass fraction $\mathrm{wt} \%$ of $\mathrm{PVC} / \mathrm{CB}$ conductive composite were studied, as shown in Fig. 1.

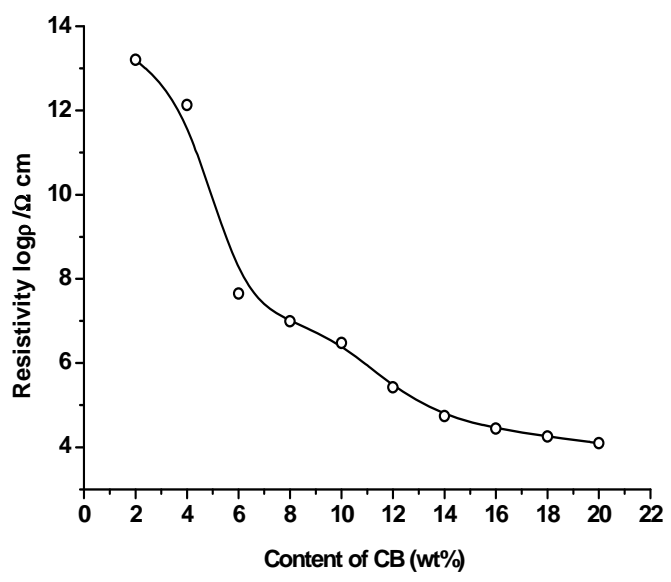

Fig. 1 Relationship of carbon black fills rate and room temperature resistivity

As can be seen from Fig. 1, the volume resistivity of the sample was gradually reduced, when carbon black filling rate increased to a characteristic value, the resistivity would dips 5 to 10 orders of magnitude, when exceeding this characteristic values, adding carbon black, a small change was in the resistivity. This phenomenon was called seepage effect (percolation), the corresponding black fill rate characteristic value was the percolation threshold.

The conductive carbon black in the composite was mainly determined by the distribution of silica particles in the resin matrix of the composite system, the electron transport problems mainly related to the distribution of the conductive filler in the matrix. When the filling amount of carbon black was low, the carbon black particles and other aggregates irregularly dispersed in the matrix, so it was difficult to form throughout the entire material of the conductive paths, in this case, the conductivity performance of the composite system depended on the PVC matrix, the composite was still in the range of insulators $(\rho>1012 \Omega \bullet \mathrm{cm})$. With the increase of carbon black loading, interfacial energy surplus of the polymer - filler was increasing, the number of conductive particles in contact with each other increased, the conductive particles began to form a conductive network, from a macro, the system resistivity $\rho$ declined rapidly. The experimental results showed that for HG-1P type super-conductive carbon black, conductive composite of the PVC / CB percolation threshold was about $10 \%$.

Relationship of Volume Resistivity and Temperature ( $\rho-t)$. As shown in Fig. 2, the performance of PVC / CB composite resistance - temperature changing was NTC behavior, in the first half of the temperature rise, the resistivity decreased linearly with temperature, but the trend was relatively flat; when the temperature was around $150 \sim 180{ }^{\circ} \mathrm{C}$, a clear downward trended, it was showing the switching characteristics. 


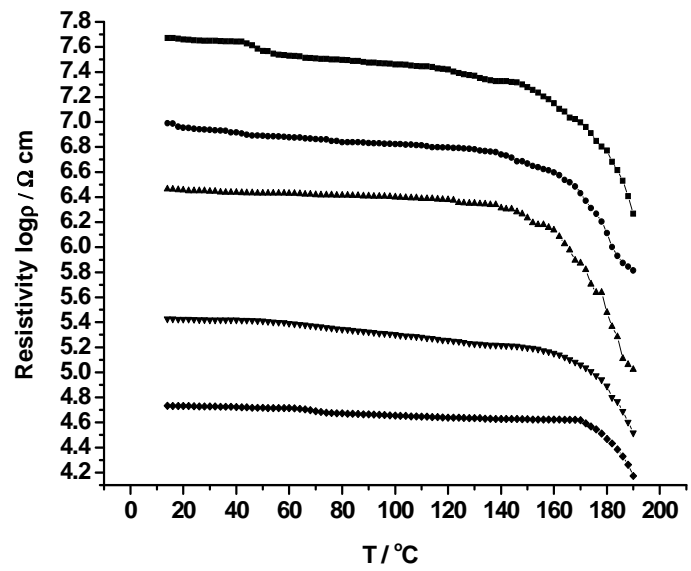

Fig. 2 PVC / CB composite resistance temperature characteristic curves (from top to bottom, the carbon black content was $6 \%, 8 \%, 10 \%, 12 \%, 14 \%$ )

Why there will be the NTC phenomenon, mainly the following reasons: PVC is the polar polymer, in PVC macromolecular chains there is a stronger electron-withdrawing groups - $\mathrm{Cl}$, a lot of polar groups were around the huge fused aromatic ring of the carbon black. When the silica particles were filled into the PVC matrix, the electrostatic adsorption was happened between these groups and $-\mathrm{Cl}$, so that the active electronics in the surface of the carbon black particles was in a frozen state. Therefore, the contribution of this part was very small particles to macroscopic resistance. In the process of temperature rise, frozen state of the electrons was gradually absorbed energy, but not elevated to a sufficiently high temperature $\left(150^{\circ} \mathrm{C}\right.$ to $\left.180^{\circ} \mathrm{C}\right)$, the absorbed energy was not sufficient to make these electronic re-released to participate in the migration process, when the temperature rises to a certain level (at $150^{\circ} \mathrm{C}$ to $180^{\circ} \mathrm{C}$ ), the absorbed energy was strong enough, a large number of these freeze electronic freed, leading the tunneling effect constantly enhance. Thus, the resistance decreased with increasing temperature.

PVC / CB Conductive Composite Section Morphology. The cross-section analysis of the carbon black content of 10\% PVC / CB composite was illustrated in Fig. 3. As can be seen from the SEM photograph, the morphology of the cross-sectional was hilly shape and some layer sheet structure, the dark area was a carbon black rich region, and the dark area was a carbon black rich region, the dark and light areas was relatively evenly distributed, the dispersion effect of the carbon black was better.

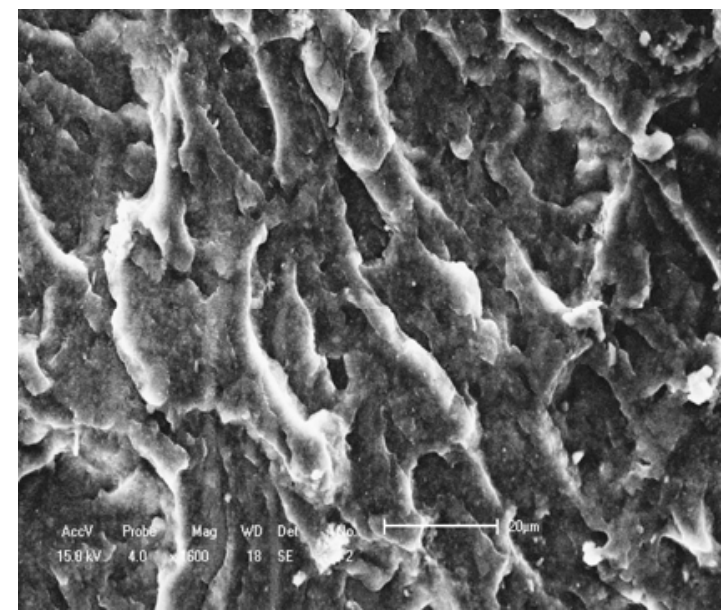

Fig.3 SEM of sample cross-section

\section{Conclusions}


(1) The volume resistivity was reduced, when carbon black filling rate increased to a characteristic value, the resistivity reached 5 to 10 orders of magnitude, when exceeding this characteristic values, adding carbon black, a small change was in the resistivity.

(2) The resistance - temperature changing of composite was NTC behavior, in the first, with temperature rise, the resistivity decreased linearly, but the trend was relatively flat; when the temperature was around $150 \sim 180{ }^{\circ} \mathrm{C}$, it was the switching characteristics.

(3) From the SEM photograph of composite, the dark and light areas were relatively distributed, the dispersion effect of the carbon black was good.

\section{Acknowledgements}

This work was financially supported by the Jilin Agricultural University Scientific Research Foundation.

\section{References}

[1] X. P. Bi, Q. C. Ying, R. Y. Qian, Macromol. Chem. Vol. 193(1992), p. 2905.

[2] G. Q. Shi, S. Jin, G. Xue, Science Vol. 267(1995), p. 994.

[3] J. C. Scott, P. Pfluger, M. T. Krounbi, Phys. Rev. B Vol. 28(1983), p. 2140.

[4] Azim S. Syed, Satheesh A., Ramu, K.K., et al., Progress in Organic Coatings Vol. 55(2006), p. 1.

[5] P. Sheng, E. K. Sichel, J. I. Gittleman, Phys. Rev. Lett. Vol. 40(1978), p. 1197. 